\title{
Editorial: Inaugural Message
}

In the field of health sciences, research based work is progressing day by day and need to accredit and publish original work of authors is addressed in many ways. We have tried to lay a foundation stone and a platform to serve and help medical and health sciences research accomplishments in Pakistan and abroad, following current international guidelines in the publishing field.

In order to facilitate the original research work, National Journal of Health Science (NJHS) is established under the umbrella of NIBD publications that will address: significant and latest development in Health Sciences and Life Sciences, academic research and inspiration to improve practice, provision of online journals and print publications to individuals and institutions and creating public access through our journal by conducting research methodology workshops for researchers to help them prepare research papers and get them published.

Quarterly hosting different scholarly manuscripts and latest findings related to broad spectrum of research disciplines for e.g. microbiology, molecular biology, biochemistry, pharmacy, general surgery, general medicine, public health, pediatrics, pathology, dentistry, biotechnology also includes other disciplines. NJHS is offering, a well-organized and user friendly online handling system. Moreover, we will ensure publication of high quality manuscripts via efficient peer review process that involves two independent reviewers and the process will be double blind for the reviewer and the author as well.

NJHS aims to provide constructive, substantial and reliable involvement of the scientific community. Our vision is to propel a transformational initiative, by providing dedicated services to medical profession to publish research based knowledge applicable to the best medical practices.

NJHS would like to acknowledge persons involved in the establishment of NJHS, it's planning, organization, formulation of mission and vision statement, the editorial board and professional staff members. NJHS would like to thank and express gratitude to the people who got involved in this journal from the authors, reviewers and editorial staff whose sincere hard work has made it reality. We look forward for its positive growth by the help and encouragement of its well wishers and contributors that trusted us from the beginning.

NJHS team is certain that with this unconditional support from such a prominent and large team of bio-researchers, doctors, students and healthcare professionals who wish to be kept informed and up-to-date with the latest and most important developments in the field of Health Sciences, will definitely become one of the most prestigious journal.

doi.org/10.21089/njhs.11.0001

Editor

Amna Yahya

NJHS

16, Maqbool Society, Block 7-8, Lal Muhammad Ch. Road, Karachi - 75350

Web:www.njhsciences.com 